\title{
Aportaciones del área de educación física a la competencia básica «AprenderaAprender» \\ Contributions of the physical education area of core competence «Learning to learn»
}

\author{
*Dolors Cañabate Ortíz, ** $\mathrm{M}^{\mathrm{a}}$ Luisa Zagalaz \\ *Universidad de Girona **Universidad Jaén
}

\begin{abstract}
Resumen: Ante un momento de cambio en la educación, con la entrada en vigor de la LOE (Ley Orgánica de Educación, 2006), el objetivo de este artículo es enfrentarse con el reto de tratar de responder a la necesidad de concretar aprendizajes de acuerdo con el nuevo currículum basado en competencias. El presente artículo surge del interés de poder ofrecer un enfoque que facilite el planteamiento de las unidades didácticas desde el área de educación física en relación con los principios pedagógicos de la LOE. Partiendo de este compromiso, presentamos una propuesta de las aportaciones de la educación física a la Competencia Básica Aprender a aprender lo cual implica desarrollar una serie de capacidades en función de saber realizar habilidades.
\end{abstract}

Palabra clave: Didáctica, educación física, competencias, capacidades, habilidades, Aprender a aprender.

Abstract: In front of a moment of change in the education, with the entry into force of the LOE (Ley Orgánica de Educación, 2006), the aim of this article is to face the challenge of trying to answer to the need to make concrete learning agreements with the new curriculum based on competences. The present article arises from the interest of being able to offer an approach that facilitates the exposition to show the didactic units from the area of physical education in relation with the pedagogic principles of the LOE. Departing from this commitment, let's sense beforehand an offer of the contributions of the Physical Education to the Basic Competence to learn to learn which entails to develop some capacities in fuction of knowing to do skills.

Key words: Didactics, physical education, competences, capacities, skills, learn to learn.

\section{Introducción}

La entrada en vigor de la LOE (Ley Orgánica de Educación, 2006) exige a los profesionales dela educación a adaptarse a los nuevos enfoques y retos que comportan las directrices de la nueva ley. Una delas novedades del nuevo marco educativo es la introducción de las competencias básicas, quedando reflejado en el mismo Preámbulo de la Ley.

Las competencias son un referente para la acción educativa y nos informan sobre lo que debemos ayudar al alumnado a construir, adquirir y desarrollar, así como también un referente para la evaluación.

En el ámbito de la educación una competencia consiste en una intervención eficaz en los diferentes ámbitos de la vida a través de acciones en las que semovilizan al mismo tiempo y demanera relacionada, componentes actitudinales, procedimentales y conceptuales.

El concepto de competencia es un elemento ya apuntado por el aprendizaje significativo, la necesidad de funcionalidad de los aprendizajes supone la condición de integrar los diferentes saberes y hacer frente desde las diferentes áreas.

Una competencia no es una simple habilidad para hacer cosas sino para resolver problemas y esto quiere decir conocer para comprender, tener capacidad de resolución para actuar y tener una actitud, un compromiso (Serramona, 2007)

El Real Decreto 1513/2006, que establece los aspectos básicos del currículum, define competencia como «la capacidad de utilizar los conocimientos y habilidades, de manera transversal e interactiva, en contextos y situaciones que requieren la intervención de conocimientos vinculados a diferentes saberes, cosa que implica la comprensión, la reflexión y el discernimiento teniendo en cuenta la dimensión social de cada situación»

Enseñar por competencias significa enseñar conocimientos (saber), capacidades (saber hacer), habilidades (saber ser), actitudes (saber estar), acciones (saber actuar). Es decir, educar personas que tengan conocimientos sobreel mundo(saber); que dispongan delos instrumentos

Fecha recepción: 28-09-09 - Fecha envío revisores: 19-10-09 - Fecha de aceptación: 02-06-10 Correspondencia: Dolors Cañabate Ortíz

Facultad de Educación y Psicología.

C/ Emili Grahit, 77. 17071 Girona

E-mail: dolors.canyabate@udg.edu para comprenderlos (saber hacer), y para que puedan habitarloy mejorarlo (saber ser y saber estar).

\section{Justificación}

La educación física puede y debe promover aprendizajes relevantes que ninguna otra asignatura es capaz de realzar, tanto a nivel personal como social.

«La finalidad de la educación física es preparar a los niños y a los jóvenes para una participación gratificante en la cultura del movimiento a lo largo de toda la vida, dedicando también especial atención a la promocióny adquisición de estilos de vida activos i saludables» (Carreiro, 2009, p. 8).

La educación física pretende el desarrollo de la persona en su globalidad, lo que implica aspectos motrices, cognitivos, efectivos y sociales. Bajo esta perspectiva tiene mucho que aportar a las competencias básicas del currículum.

El proceso de secuenciación que se presenta en este artículo ha sido contrastado con otros docentes que comparten el interés por llevar a cabo una educación física desde una perspectiva crítica, reflexiva y útil para la vida, colaborando así en el desarrollo integral de las personas. La propuesta que aquí se presenta, quiere poner el acento de su desarrollo en la competencia básica Aprender a aprender.

\section{Marco teórico}

Cuando se habla de competencias, hay toda una teoría psicológica, pedagógica y sociológica detrás que, subrayando la necesidad de significación en todo aprendizaje, entiende el hecho de hacerse competente como un proceso de aprendizajes globales que integran contenidos de diferentes tipos (conocimientos, habilidades y actitudes). Aprendizajes que comprenden el desarrollo de capacidades más que la exclusiva adquisición de contenidos puntuales y descontextualizados. La corriente de las competencias básicas implica la búsqueda de aquello que es esencial para ser aprendido. Se trata de seleccionar aquellas capacidades que, de alguna manera, se consideren realmente indispensables para facilitar la plena realización personal y social. (Noguera, 2000, p. 1) 
Las competencias son una nueva forma de enfocar la educación. Entendiendo por competencia la capacidad para poder resolver problemas complejos utilizando conocimientos, habilidades y actitudes. Pone énfasis en la habilidad y, por tanto, es una habilidad práctica.

La formación de una competencia permite a las personas que pongan en movimiento, apliquen e integren los conocimientos que han adquirido en situaciones diversas, complejas e impredecibles, definiéndola como «la capacidad de actuar eficazmente en un número determinado de situaciones, capacidad basada en los conocimientos pero que no se limita a ellos» (Perrenoud, 1999, p. 7).

Las experiencias sobre formación en competencias básicas se iniciaron en Cataluña con el estudio efectuado entre 1998 y 1999 por el Dr. Jaume Sarramona Lópezen coordinación con la FREREF(Federación de Regiones Europeas para la Investigación, la Educacióny la Formación) y el Departament d'Ensenyament (Departamento de Enseñanza) de la Generalitat. En dicha investigación, a partir de un listado de competencias estructurado en relación a las áreas del currículum, se efectuó una amplia consulta social y profesional cuyo resultado fue la elaboración de un listado de competencias básicas que se dio a conocer a los centros escolares y que sirvióa la administración educativa para iniciar procesos de evaluación interna sobre su dominio.

Según autores como Barahona, Campos, Pérez, Guerras, Casado, Feltrer, Iranzo, Bilbao (2008), el área de educación física contribuye, a la competencia de Aprender a Aprender mediante el conocimiento de sí mismo y de las propias posibilidades y carencias como punto de partida del aprendizaje motor desarrollando un repertorio variado que facilite su transferencia a tareas motrices más complejas. Ello permite el establecimiento de metas alcanzables cuya consecución genera autoconfianza. Al mismo tiempo, los proyectos comunes en actividades físicas colectivas facilitan adquirir recursos de cooperación.

A través del enfoque metodológico que se le da al área de educación física, la competencia Aprender a Aprender se encuentra en estrecha relación con la competencia autonomía einiciativa personal, favoreciendo la toma de conciencia sobre los efectos que la actividad física tiene en la salud individual y colectiva, propiciando la reflexión sobre los cambios de nuestro cuerpo a lo largo de la vida, así como los efectos beneficiosos o perjudiciales que tienen determinadas prácticas sobre la salud. Gómez, Rivera, Fernández, Gorrín, Pacheco y Sosa(2008).

\section{Objetivos}

Este artículo pretende plantear la realidad curricular introduciendo algunos matices y perspectivas que pueden ayudar a la hora de construir los propios argumentos en la programación por competencias desde elárea de educación física. Para ello nos hemos centrado en la competencia Aprender a Aprender.

Debemos tener en cuenta como siempre que todos los planteamientos teóricos dependen de cómo se apliquen a la práctica.

El objetivo principal es: Presentar una propuesta de secuenciación didáctica en relación a las aportaciones del área de educación física a la competencia básica Aprender a Aprender. requiere:

La concreción de este objetivo

\begin{tabular}{|c|c|}
\hline Capacidades & Habilidades \\
\hline $\begin{array}{l}\text { CONOCER LAS } \\
\text { PROPIAS } \\
\text { CAPACIDADES }\end{array}$ & $\begin{array}{l}\text { - Saber autoevaluarse. } \\
\text {-Identificar las potencialidades y dificultades. } \\
\text {-Adquirir conciencia sobre el comportamiento y los sentimientos que } \\
\text { generan los procesos de aprendizaje en uno mismo. } \\
\text {-Ser consciente sobre qué me pasa / qué hago delante de una dificultad. }\end{array}$ \\
\hline $\begin{array}{l}\text { REFLEXIONAR SOBRE } \\
\text { CÓMO APRENDER }\end{array}$ & $\begin{array}{l}\text { - Descubrir actitudes o condiciones necesarias para aprender (atención, concentración, memoria, comprensión, expresión...) } \\
\text { - Descubrir actitudes o condiciones que dificulten el aprendizaje. } \\
\text {-Identificar el proceso básico para resolver tareas (¿qué me piden?, ¿qué sé?, ¿qué necesito saber?, ¿cómo lo puedo hacer?) } \\
\text {-Descubrir formas para aprender cooperativamente. }\end{array}$ \\
\hline $\begin{array}{l}\text { ADQUIRIR } \\
\text { ESTRATEGIAS PARA } \\
\text { DESARROLLAR LAS } \\
\text { PROPIAS } \\
\text { CAPACIDADES }\end{array}$ & $\begin{array}{l}\text {-Identificar y ejercitar las propias capacidades } \\
\text {-Ejercitar la memoria a corto, medio y a largo término utilizando diferentes técnicas. } \\
\text {-Resolver actividades que requieran diferentes grados de tensión y concentración. } \\
\text {-Poner en práctica diferentes estrategias nuevas y creativas buscando, recuperando y tratando la información. } \\
\text {-Poner en práctica diferentes estrategias de comunicación escrita, verbal y no verbal } \\
\text { - Resolver actividades mediante trabajo cooperativo. }\end{array}$ \\
\hline $\begin{array}{l}\text { MEJORAR LA } \\
\text { AUTOESTIMA Y EL } \\
\text { SENTIMIENTO DE } \\
\text { COMPETENCIA }\end{array}$ & $\begin{array}{l}\text {-Resolver actividades de dificultad gradual. } \\
\text {-Recoger las actividades que se han resuelto correctamente cada día. Intercambiar valoraciones positivas entre los } \\
\text { compañeros y compañeras. } \\
\text {-Trabajar cooperativamente. }\end{array}$ \\
\hline $\begin{array}{l}\text { UTILIZAR DIFERENTS } \\
\text { MÉTODOS Y } \\
\text { ESTRATEGIAS } \\
\text { DURANTE EL } \\
\text { APRENDIZAJE }\end{array}$ & $\begin{array}{l}\text { - Hacer planes de trabajo. } \\
\text {-Organizarse y ser ordenado con el trabajo } \\
\text {-Plantearse y redactar objetivos claros y concretos. } \\
\text {-Tomar decisiones según los diferentes contextos y cuestionarse preguntas y buscar respuestas a través de diferentes } \\
\text { fuentes. } \\
\text {-Trabajar en grupo: dialogando, cooperando, expresándose, comunicando, respetando, tolerando... } \\
\text {-Trabajar individualmente y autónomamente. }\end{array}$ \\
\hline $\begin{array}{l}\text { GESTIONAR LOS } \\
\text { RECURSOS } \\
\text { NECESARIOS }\end{array}$ & $\begin{array}{l}\text { Investigar, sintetizar y trabajar la información a través de diferentes fuentes y recursos. } \\
\text { Emitir soluciones críticas sobre los recursos utilizados. } \\
\text { Aplicar diferentes recursos durante el aprendizaje. } \\
\text { Dominar los recursos audiovisuales como fuente de obtención y de transmisión de información. }\end{array}$ \\
\hline $\begin{array}{l}\text { RELACIONAR TEORÍA } \\
\text { Y PRÁCTICA }\end{array}$ & $\begin{array}{l}\text {-Utilizar el conocimiento teórico como instrumento de reflexión para analizar el fenómeno educativo (reflexionar y teorizar } \\
\text { a partir de la práctica). } \\
\text {-Identificar los marcos explicativos para las diferentes situaciones educativas. } \\
\text {-Saber justificar y argumentar. } \\
\text { - Buscar fuentes de información relevantes } \\
\text {-Saber seleccionar información relevante } \\
\text {-Habilidad de análisis y síntesis. } \\
\text { - Actuar, reflexionar, experimentar y teorizar. } \\
\text {-Reconstruir conceptos a partir de las vivencias, de las experiencias personales, de la conciencia corporal. } \\
\text {-Del placer de actuar al placer de pensar. }\end{array}$ \\
\hline $\begin{array}{l}\text { CONOCERSE A UNO } \\
\text { MISMO }\end{array}$ & $\begin{array}{l}\text { •Conocer : } \\
\quad \text { el que uno mismo ya sabe. } \\
\quad \text { el que uno quiere aprender. } \\
\quad \text {-las propias potencialidades o capacidades. } \\
\text {-Hacer autocrítica. } \\
\text { •Emitir juicios de valor sobre uno mismo y sobre el propio trabajo. }\end{array}$ \\
\hline
\end{tabular}

· Concreción de capacidades en relación a la capacidad Aprender a Aprender.

- Relación de capacidades y habilidades de aprendizaje.

Concreción de las aportaciones del área de educación física a la competencia.

Los profesionales de la educación somos conscientes que todos los planteamientos teóricos dependen de como se apliquen a la práctica, de ahí nuestro interés de poder participar en este estudio en aportar elementos que faciliten el proceso de enseñanza y aprendizaje de competencias.

\section{Diseño metodológico}

Para realizar el objetivo principal se llevaron a cabo una serie de reuniones durante el mes de julio entre un grupo de profesionales. Los participantes fueron un profesor de didáctica de primaria de la Universidad de Girona junto con especialistas de educación física, como maestros y licenciados. Ejerciendo un trabajo cooperativo, se llegó a establecer una lista de aquellas capacidades y habilidades que contribuyese a desarrollar la competencia básicaAprender a Aprender. Esta lista es un paso previo a una futura elaboración de una programación de actividades en el aula para trabajarla.

El análisis en profundidad de este proceso particular no pretende generalizar resultados, pero debe permitir generar hipótesis y tomar decisiones respecto a la mejora del mismo (Latorre, del Rincón, Arnal, 1996), así como ofrecer un enfoque que facilite el planteamiento de las unidades didácticas desde área de educación física en relación al nuevo enfoque curricular basado en competencias. La metodología requiere la definición de un tema, un caso y un foco de estudio (Stake, 1999):

- Tema:Aportaciones de la educación física a la Competencia Básica Aprender a Aprender.

- Caso: Laprogramación en competencias desde elárea de educación física en relación con los principios pedagógicos de la LOE. 
-Foco de estudio: Secuenciación didáctica en competencias.

La propuesta que aquí se presenta parte de las siguientes premisas:

- Conocimiento de si mismo y de las propias limitaciones.

- Autosuperación personal, perseverancia y actitud positiva.

- Organización individual y colectiva

- Adquirir una buena actitud y predisposición para aprender nuevos aprendizajes.

- Conocer y aceptar las limitaciones propias y de los demás.

-Aprender a compartir, dialogar, escuchar, ayudar y pedir ayuda.

- Aprendizaje cooperativo y entre iguales.

- Desarrollo de las habilidades sociales.

Para poder desarrollar esta competencia nos planteamos que los alumnos y alumnas:

- Conozcan sus propias capacidades

- Reflexionen sobre cómo aprenden

- Adquieran estrategias para desarrollar sus propias capacidades

- Mejoren la autoestima y el sentimiento de competencia personal

Deberán ser capaces de:

- Utilizar diferentes métodos y estrategias durante el aprendizaje.

- Gestionar los recursos necesarios

- Conocerse a si mismos

- Evaluar el propio aprendizaje.

De nosotros los educadores, depende cómo planteamos una educación basada en competencias, si seremos capaces de analizarlas, de profundizar en ellas, si las aplicamos demanera adecuadaa las necesidades de nuestro alumnado, de acuerdo al contexto de nuestro centro, así como del momento educativo y de las necesidades que vivimos.

En primer lugar, se han definido algunas de las capacidades que se encuentran en relación a la competenciaAprender a Aprender. En segundo lugar hemos seguido avanzando en nuestra concreción y hemos relacionado las capacidades con habilidades, y la última parte del artículo la dedicamos a la presentación de algunas de las aportaciones que desde elárea de educación física puede ofrecer ala competencia básicaAprender a Aprender.

\section{Resultados y discusión}

A continuación vamos a dar a conocer la relación de capacidades y habilidades de acuerdo a la competencia Aprender a Aprender. Esta relación no pretende ser una propuesta cerrada y única, sino ofrecer posibilidades a la gran oferta que de ellas ofrece dicha competencia (Tabla 1)

Las competencias movilizan conocimientos, buena parte de ellos son conceptuales, pero también procedimentales y normativos. Según

A1.- Saberse autoevalua

- Identificar prácticas poco saludables.

-Inventar juegos deportivos.

- Llevar a término juegos cooperativos.
Tabla 2. Aportaciones del área de educación física a la competencia Aprender a Aprender .

- Mantener una actitud respetuosa hacia la propia realidad corporal y la de los otros.

A2.- Identificar las potencialidades $y$ las dificultades

- Adoptar roles diferentes dentro de una misma actividad física.

aquello que se puede hacer a través del trabajo en equipo y con la ayuda de los demás.

-Identificar los comportamientos y sentimientos que generan las dificultades y los éxitos.

- Anticipar posibles desenlaces delante las dificultades que presenta una actividad motriz

B1.- Descubrir actitudes o condiciones que dificultan o favorecen el aprendizaje

- Vivenciar diferentes elementos que facilitan el aprendizaje de contenidos o procesos motrices (la tensión, la relajación, la respiración, la postura, etc.).

- Identificar la actividad, las condiciones de ésta y las respuestas posibles.

- Dosificar y adecuar el nivel de esfuerzo para resolver una actividad física.

- Descubrir procedimientos o técnicas pre-deportivas específicas.

B3.- Descubrir formas para aprender cooperativamente

- Vivenciar las diferencias entre el trabajo individual y el trabajo en equipo.

Aprender diferentes técnicas de tro como una forma positiva para resolver actividades motrices en equipo.

- Experimentar el dar, el recibir y el compartir.

SIRIR ESTRATEGIAS PARA DESARROLLAR LAS PROPIAS CAPACIDADES

ercitar las propias capacidades

- Proponerse mejoras personales y de equipo a corto término.

- Rdquirir un cons diferentes modalidades y dificultades crecientes

- Ejecutar movimientos de mayor a menor dificultad con los segmentos corporales dominantes.

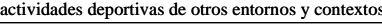

- Valorar a nivel individual y colectivo el trabajo realizado desde el punto de vista motriz

- Practicar la elaboración de bailes y coreografías sencillas.

D1.- Resolver actividades de dificultad gradua

- Realizar juegos de diferentes modalidades y dificultades crecientes.

- Adquirir un control motor y corporal de manera progresiva.

D2.- Recoger las actividades que se ha resuelto correctamente cada día

- Poner en común todo aquello que se ha trabajado durante la sesión de educación física.

-

- Utilizar un registro para recoger los éxitos individuales y colectivo.

D3.- Intercambiar valoraciones positivas entre compañeros

-

- Llevar a término masajes y estrategias de relajación entre cor los compañeros.

D4.- Trabajar a través del trabajo cooperativo

- Valorar a nivel individual y colectivo el trabajo realizado desde el punto de vista motriz

- Practicar en la elaboración de bailes y coreografías sencillas.

-Promover el uso adecuado de estrategias básicas de juego relacionadas con la cooperación y la oposición.

Jaume Graells, director general de la Educación Básica y el Bachillerato, las competencias son más que conocimientos, pero no son nada sin estos. Una gran parte de los conocimientos están vehiculados a diferentes saberes o disciplinas.

Las competencias no niegan las disciplinas sino que defienden su combinación como requisito para resolver problemas de cierto grado de complejidad. Las competencias ponen el énfasis en la utilización de que se hace de los aprendizajes, no de lo que se sabe. Serramona (2007).

También es objetivo de este artículo ofrecer algunas de las aportaciones del área de educación física a la competencia Aprender a Aprender, de acuerdo con lo que hemos presentado anteriormente(Tabla 1), exponiendo de forma más amplia las capacidades. Así pues, cada una de dichas capacidades se desglosa en otras más concretas, cuyas implicaciones a nivel didáctico también se especifican. Esto representa un nivel de concreción más elevado y, por ello, se presenta en una segunda tabla (Tabla 2).

Las competencias son un referente para la acción educativa y nos informan sobre lo que debemos ayudar al alumnado a construir, a adquirir y desarrollar; y también en consecuencia, un referente para la evaluación, 
útil para comprobar el nivel de logro alcanzado por los alumnos y alumnas. Sin embargo las competencias, como las capacidades no son directamente evaluables. Hay que elegir los contenidos más adecuados para trabajarlas y desarrollarlas, definir la secuencia y el grado propio de los diferentes niveles y cursos, establecer indicadores precisos de logro, y acertar en las tareas que finalmente se le pida al alumno que realice. La posibilidad de «perder el hilo» en este complejo recorrido es sin duda muy grande (Martín y Coll, 2003).

\section{Conclusiones}

Centrar la actividad escolar en la adquisición de competencias en vez de contenidos culturales y científicos no es desde luego nada fácil, especialmente por lo arduo que resulta luchar contra una inercia que no ha hecho sino incrementarse y que todavía hoy encuentra apoyos incluso en aras de mejorar la calidad.

La incorporación de las competencias básicas supone un enriquecimiento del modelo actual de currículo. De acuerdo con lo dispuesto en la LOE, las competencias básicas forman parte de las enseñanzas mínimas de la educación obligatoria, junto con los objetivos de cada área o materia, los contenidos y los criterios de evaluación. Por lo tanto, no sustituyen a los elementos que actualmente se contemplan en el currículo, sino que los completan planteando un enfoque integrado e integrador de todo el currículo escolar.

Hacer hincapié en las competencias básicas exige orientar los aprendizajes para conseguir que los alumnos desarrollen diversas formas de actuación y adquieran la capacidad de enfrentarse a situaciones nuevas.

La principal contribución de las competencias básicas consiste en orientar la enseñanza, al permitir identificar los contenidos y los criterios de evaluación. Para ello es imprescindible conocer en profundidad cada una de las competencias, así como las capacidades relacionadas, las habilidades, las destrezas... Todo este conocimiento facilitará al profesorado su intervención en el aulay por consiguiente la transferencia adecuada de las competencias en el proceso de enseñanza y aprendizaje.

\section{Bibliográfia}

Ausubel, D. O. (2002). Adquisición y retención del conocimiento. Una perspectiva cognitiva. Barcelona: Paidós.

Ausubel, D. P., Novak, J. D. \& Hanesian, H. (1978): Educational Psychology. Nueva Cork. Holt, Rinehart etwiston. Trad. Cast: Psicología educative. México: Trillas.

Barahona, J., Campos, M., Pérez, C., Guerras, A., Casado, M. V., Feltrer, J., et al. (2008). El desarrollo de las competencias básicas a través de la Educación Físca. EFdeportes Revista Digital, 118. Disponible en http://www.efdeportes.com/ [Consulta: 2009, 2 de mayo]

Chinchilla, J. L. \& Zagalaz, M. L. (2002). Didáctica de la educación física. Granada. CCS.

Carreiro da Silva, F. (2009). La gestión del currículo a través de competéncias:un enfoque desde el contexto portugués. Tándem. Didáctica de la educación física, 29, 8-9.

Coll, C. (2008). Las competencias en la educación escolar: algo más que una moda y mucho menos que un remedio. Aula de Innovación Educativa, 161, 34-39.

Comisión Europea (2004). Competencias clave para un aprendizaje a lo largo de la vida. Un marco de referencia europeo. Puesta en práctica del programa de trabajo Educación y formación 2010. Grupo de trabajo B. Competencias Clave. Comisión Europea. Dirección General deEducación y cultura. Disponible en http://www.educastur.princast.es/ info/calidad/indicadores/doc/comisión_europea.pdf. [Consulta: 2009, 16 de abril].

Contreras, O., Gutiérrez, D., García López, L. M. (2002). La fuerza del currículum de la Educación física escolar. Un análisis del papel de la fuerza en el vital actual desde la perspectiva del desarrollo. Retos. Nuevas Tendencias en Educación física, Deportes y Recreación, 1, 37-41.
Departament d'Ensenyament de la Generalitat de Catalunya. Disponible en http://www.gencat.net/educacio/csda/actuacions/est_fin/docs/ relacio_cb.pdf.[Con-sulta: 2009, 25 de febrero]

Fraile, A. (2004). El profesor de Educación física como investigador de su práctica. Tándem. Didáctica de la Educación física, 15, 37-49.

Fraile,A. \& Hernández, J. L. (2006). La formación del maestro de educación física en la encrucijada entre los marcos legislativos y las necesidades sociales. Aula de Innovación educativa, 157, 65-71.

Gómez, A., Rivera, L.J., Fernández, J.M., Gorrín, A., Pacheco, J.J. y Sosa, G.(2008). Nueva propuesta curricular para el área de Educación física en la Educación Primaria. Revista Internacional de Medicina y Ciencias de la Actividad Física y el Deporte, vol. 8, 29, 93-108

Hutmacher, W. (2003). Definición de las competencias básicas. La situación en Europa. Conference. Congrés de competències básiques. Disponible en: http://www.xtec.es/serveis/crp/a8930006/cb0.htm. [Consulta: 2009, 23 de mayo]

Jiménez, J.R. (2007). Competencias Básicas. Redes, 1, 1.

Latorre, A., Del Rincón, D. \& Arnal, J. (1996). Bases metodológicas de la investigación educativa. Barcelona: Hurtado Ediciones.

LLeixà, T. (2007). Educación física y competencias básicas. Contribución del área a la adquisición de las competencias básicas del currículo. Tándem. Didáctica de la Educación física, 23, 6-15.

Lleixà, T. (2008). Educació Física, una mirada cap al futur. Perspectiva Escolar, 321, 2-8.

LOE. Ley Orgánica 2/2006, de 3 de mayo, de Educación.

Noguera Arrom, J. (2000). Competencies Basiques per l’Educació Primária. En «L'Educació Primaria». Generalitat de Catalunya. Consell Escolar de Catalunya. Barcelona, p. 1.

Martín, E. \& Coll, C. (2003): Aprender contenidos, desarrollar capacidades. Intenciones educativas y planificación de la enseñanza. Barcelona: Edebé.

Muñoz, J. C. (2007). La Educación física en la Ley Orgánica de Educación EFdeportes Revista Digital, 105. Disponible en http:// www.efdeportes.com/ [Consulta: 2009, 25 de mayo]

OCDE (2005). The definition and selection of keycompetencies. Executive summary. Disponible en: http://www.oecd.org/dataoecd/47/61/ 35070367.pdf. [Consulta: 2009, 13 de marzo]

Parlamento Europeo y Consejo de la Unión Europea (2006). Recomendacion del Parlamento Europeo y del Consejo de 18 de diciembre del 2006 sobre las competencias clave para el aprendizaje permanente. Diario Oficial de la Unión europea. L394/10-18. 30 de diciembre del 2006.

Perremoud, P. (1999). Construir as competencias desde a Escola, 7-24. Porto alegre (Brasil): Artmed Editora.

Perrenoud, P. (2004). Diez nuevas competencias para enseñar. Barcelona: Graó.

Real Decreto 1513/2006, de 7 de diciembre, por el que se establece las enseñanzas mínimas de la Educación Primaria. BOE nº 293 (2006).

Ruíz Pérez, L. M. (2004). Competencia motriz, problemas de coordinación y deporte. Revista de educación, 335, 21-23.

Stake, R. E. (1999). Investigación con estudio de casos. Madrid: Morata.

Rychen, D. S. (2003). La naturaleza de las competencias clave. Una perspectiva interdisciplinaria e internacional. Congrés de competències básiques. Disponible en: http://www.xtec.es/serveis/crp/a8930006/ cb0.htm [Consulta: 2009, 15 de marzo].

Serramona, J. (2003). L’aportació de Catalunya a la identificació, gradació i avaluació de les competències bàsiques. Congrés de competències básiques. Disponible en: http://www.xtec.es/serveis/crp/a8930006/ cb0.htm. [Consulta: 2009, 15 de marzo].

Serramona, J. (2004). Las competencias básicas en la Educación Obligatoria. Barcelona CEAC.

Serramona, J. (2007). Entrevista . Escola Catalana, 42, 30-36.

Serramona, J. \& López, L. (2000). Identificació de les Competències Bàsiques en l'Ensenyament Obligatori. Barcelona: Generalitat de Catalunya: Departament d'Ensenyament.

Vaca Escrivano, M. (2002). Reflexiones en torno a las competencias profesionales solicitadas a los maestros de educación física. Retos. Nuevas tendencias en Educación física y Recreación.

Zabala, A. \& Arnau, C. (2007). 11 Ideas claves: como aprender y enseñar competencias. Barcelona: Graó. 